\title{
VIVÊNCIAS DA DELIBERAÇÃO MORAL DE ENFERMEIRAS NO ATENDIMENTO \\ PRÉ-HOSPITALAR MÓVEL
}

\author{
EXPERIENCES OF THE MORAL DELIBERATION OF \\ NURSES IN MOBILE PRE-HOSPITAL CARE
}

\section{EXPERIENCIAS DE LA DELIBERACIÓN MORAL DE LAS ENFERMERAS EN LA ATENCIÓN MÓVIL PRE-HOSPITALARIA}

\author{
Simone da Silva Oliveira ${ }^{1}$ \\ Adriana Braitt Lima ${ }^{2}$ \\ Darci de Oliveira Santa Rosa ${ }^{3}$ \\ Genival Fernandes de Freitas ${ }^{4}$ \\ Mariana Oliveira Antunes Ferraz
} Como citar este Artigo: Oliveira SS, Lima AB, Santa Rosa DO, Freitas GF, Ferraz MOA. Vivências da deliberação
moral de enfermeiras no atendimento pré-hospitalar móvel. Rev baiana enferm. 2021;35:e38733.

Objetivo: compreender como as enfermeiras vivenciam a ação de deliberação moral no cenário de prática. Método: pesquisa fenomenológica fundamentada no referencial de Alfred Schutz realizada mediante entrevistas com 12 enfermeiras de um Serviço de Atendimento Móvel de Urgência. Da análise ideográfica e nomotética foram obtidas as categorias concretas do vivido. Resultados: emergiram três categorias: significados da ação da deliberação moral das enfermeiras no atendimento pré-hospitalar móvel de urgência que se referem ao contexto do atendimento; o conhecimento técnico-científico como fundamento para a ação da deliberação moral; e a dimensão social das relações estabelecidas entre as equipes como fonte de problemas éticos. Conclusão: foram desvelados fatores que incidem na prática, revelando elementos que favorecem a deliberação, como as experiências do vivido, o compartilhamento das situações e os conflitos que demandam a tomada de decisão. Emerge, assim, um novo olhar para a prática das enfermeiras pautada em um agir ético, responsável e prudente.

Descritores: Bioética. Enfermagem. Enfermeiras e Enfermeiros. Resolução de Problemas. Socorro de Urgência.

Objective: to understand how nurses experience the action of moral deliberation in the practice scenario. Method: phenomenological research based on Alfred Schutz's reference conducted through interviews with 12 nurses from a Mobile Urgency Care Service. From the idiographic and nomothetic analysis, the concrete categories of the lived were obtained. Results: three categories emerged: meanings of the action of nurses' moral deliberation in the mobile pre-hospital emergency care that refer to the care context; technical-scientific knowledge as the basis for the action of moral deliberation; and the social dimension of the relationships established between the teams as a source of

\footnotetext{
Enfermeira. Mestra em Enfermagem. Enfermeira do Serviço de Atendimento Móvel de Urgência da Microrregião Alagoinhas. Alagoinhas, Bahia, Brasil. simone192samu@gmail.com. http://orcid.org/0000-0003-1884-7026.

Enfermeira. Doutora em Enfermagem. Docente da Universidade Estadual de Feira de Santana. Feira de Santana, Bahia, Brasil. http://orcid.org/0000-000 I-7893-9753.

3 Enfermeira. Pós-doutora em Bioética. Docente do Programa de Pós-Graduação da Universidade Federal da Bahia. Salvador, Bahia, Brasil. http://orcid.org/00000002-565।-2916.

4 Enfermeiro. Pós-doutor em História e Antropologia dos Cuidados. Docente da Escola de Enfermagem da Universidade de São Paulo. São Paulo, São Paulo, Brasil. http://orcid.org/0000-0003-4922-7858.

5 Enfermeira. Mestra em Ciências da Saúde. Docente da Universidade Estadual do Sudoeste da Bahia. Jequié, Bahia, Brasil. http://orcid.org/0000-0002-7328-6025.
} 
ethical problems. Conclusion: factors that affect practice were revealed, unveiling elements that favor deliberation, such as experiences of the lived, the sharing of situations and conflicts that require decision-making. Thus, a new look emerges for the practice of nurses based on an ethical, responsible and prudent action.

Descriptors: Bioethics. Nursing. Nurses. Problem Solving. Emergency Relief.

Objetivo: entender cómo las enfermeras experimentan la acción de la deliberación moral en el escenario de práctica. Método: investigación fenomenológica basada en la referencia de Alfred Schutz realizada a través de entrevistas con 12 enfermeras de un Servicio de Atención Móvil de Urgencia. A partir del análisis ideográfico y nomotético, se obtuvieron las categorías concretas de los vividos. Resultados: surgieron tres categorías: significados de la acción de la deliberación moral de las enfermeras en la atención pre-hospitalaria móvil de urgencia que se refieren al contexto de la atención; conocimiento técnico-científico como base para la acción de la deliberación moral; y la dimensión social de las relaciones establecidas entre los equipos como fuente de problemas éticos. Conclusión: se revelaron factores que afectan a la práctica, revelando elementos que favorecen la deliberación, como las experiencias de los vividos, el intercambio de situaciones y conflictos que requieren la toma de decisiones. Así, surge un nuevo aspecto para la práctica de las enfermeras basada en una acción ética, responsable y prudente.

Descriptores: Bioética. Enfermería. Enfermeras y Enfermeros. Solución de Problemas. Socorro de Urgencia.

\section{Introdução}

O cenário brasileiro da Rede de Atenção às Urgências e Emergências tem, entre os seus componentes, o Serviço de Atendimento Móvel de Urgência (SAMU) como um serviço de utilidade pública, com o intuito de assegurar o direito de acesso ao serviço de saúde pelo usuário, por meio de ligação telefônica e acolhimento dos chamados por uma Central de Regulação das Urgências. A equipe de atendimento conta com profissionais habilitados, como o Médico Regulador que atua na dinâmica do serviço, classificando o agravo e definindo o tipo de Unidade Móvel para cada caso, bem como as equipes de tripulação composta por outros profissionais ${ }^{(1)}$, dentre os quais destacam-se as enfermeiras.

No tocante à prática das enfermeiras neste serviço de Atendimento Pré-hospitalar Móvel (APHM), torna-se um desafio constante a preocupação com o agir ético do profissional responsável, pautado em competências que ajudem nas tomadas de decisões em circunstâncias adversas, inusitadas e conflituosas, distintas das que ocorrem no ambiente hospitalar ${ }^{(2)}$. Frente a esse fenômeno, é preciso que a enfermeira desenvolva a competência ética na prestação dos cuidados, tendo em vista que essas experiências exigem atualizações dos atendimentos às normas e deveres, que podem envolver tanto a pessoa doente como também os familiares e outros membros da equipe multiprofissional. Nesse contexto, as tensões entre valores e deveres profissionais podem ser vivenciadas e comprometer a integridade moral das enfermeiras ${ }^{(3)}$, sendo, portanto, imprescindível, reconhecer os significados dos atos de deliberar dessas profissionais, que mantêm uma rede de relação interpessoal no espaço do atendimento.

Então, considera-se a ação de deliberar sobre um conflito ético, o processo de maturação das decisões fazendo imperar um curso de ação prudente e responsável. Torna-se necessária a dúvida, a ocorrência de tensionamento entre os valores e os deveres individuais ou coletivo no momento do agir, para fazer emergir a prática da Bioética Deliberativa ${ }^{(4)}$.

Assim, em uma perspectiva da Fenomenologia Social, esse mundo-vida, contextualizado pela intersubjetividade de um grupo social de enfermeiras que abarca as suas vivências cotidianas, mostra-se com a necessidade de expressar atitudes em situações concretas que suscitam enfrentamentos, em um tempo de dúvidas, questionamentos ou necessidade de correções de elementos da prática cotidiana desses profissionais. Essas inquietações envolvem a atuação da enfermeira na experiência moral, com uma busca frequente para o saber agir de modo valorativo e responsável ${ }^{(5)}$. 
Nesse sentido, Schutz discute a estrutura social e expressa ser a relação social um elemento fundamental na interpretação dos significados, elege como relevante a compreensão do cotidiano e a existência humana no mundo da vida, também denominado mundo social, mundo cotidiano e mundo do senso comum ${ }^{(6)}$. É nesse mundo que se abrem as experiências éticas.

No momento do atendimento primário, que ocorre na rua, a ação intencionada para um socorro adequado segue um trilhar que condiciona a necessidade de pensamento crítico dos profissionais frente às práticas cotidianas e às questões éticas dirigidas para uma realidade social que se tem familiaridade por meio de uma convivência prévia, experienciada no mundo cotidiano do APHM.

Estudo sobre questões éticas nos atendimentos de enfermeiras de serviços de ambulância, realizado nos Estados Unidos, desvelou que uma convivência com a imprevisibilidade, muitas vezes ameaçadora à vida, torna as tomadas de decisões éticas desafiantes, caracterizando a atuação das profissionais como uma tarefa complexa, difícil, carregada de responsabilidade ${ }^{(7)}$.

Ciente da complexidade das ações de cuidados implicadas no fazer da enfermeira no cenário do APHM, a deliberação moral torna-se um requisito essencial ao ampliar a competência ética e a efetividade das relações na tomada de decisão.

Diante desse cenário, na perspectiva de que as enfermeiras que atuam no APHM são um grupo social que lida com vidas, dirige suas ações práticas e éticas para todas as pessoas envolvidas no momento do cuidado, propõe-se o estabelecimento de estratégias para a tomada de decisões, com vistas à minimização de danos frente aos problemas éticos que podem emergir ${ }^{(8)}$. Assim, fez-se o seguinte questionamento: "Como as enfermeiras do APHM vivenciam a ação da deliberação moral no seu cotidiano?"

O estudo do fenômeno da deliberação moral possibilitará reflexões sobre as experiências morais e éticas por enfermeiras, frente aos problemas éticos no seu cotidiano, às relações sociais e a dimensão de cuidado, em um ambiente de trabalho marcado por limitações e situações adversas encontradas no atendimento em rua.

Ciente do mundo de relações, da comunicação existente no APHM e que o significado e sentido da ação de deliberar estão atribuídos a vivências cotidianas do fenômeno, o objetivo deste estudo foi compreender como as enfermeiras vivenciam a ação de deliberação moral no seu cenário de prática.

\section{Método}

Trata-se de estudo fenomenológico sobre a deliberação, realizado no período de janeiro a março de 2014. A Fenomenologia Social interessa-se não pelos atos singulares, nem os comportamentos individuais, fechados numa consciência de si, mas a compreensão do que constitui um determinado grupo social que vive uma situação típica. O mundo cotidiano é comum a todos e não se resume a um mundo individual, mas intersubjetivo, que se compartilha com os nossos semelhantes ${ }^{(9)}$.

A Fenomenologia Social, de Alfred Schutz, foi escolhida para este estudo porque proporciona compreensão de questões que expressam motivação, que fundamenta e impulsiona a ação desenvolvida no mundo de suas relações sociais na prática cotidiana dos que compartilham projetos de vida ${ }^{(6)}$. Assim, este estudo teve como cenário as bases descentralizadas de um SAMU Metropolitano, por considerar que este serviço disponibiliza para os seus usuários um atendimento com escuta médica por 24 horas, atrelado a uma Central de Regulação das Urgências que retrata o mundo-vida do APHM predominante no cenário brasileiro.

Intencionalmente, as participantes foram 12 enfermeiras que atenderam os critérios de inclusão: ser enfermeira que intervém junto à equipe nas Unidades Móveis, ter vivenciado no seu cotidiano a deliberação moral no processo do cuidar em um Serviço de Atendimento Móvel de Urgência e ter no mínimo seis meses de atuação neste serviço. Os critérios de exclusão foram: ser enfermeira com exercício de cargo administrativo e estar afastada da assistência por qualquer motivo. 
Para garantir o anonimato, os participantes foram identificados por codinomes que representam palavras que, na origem do significado, estão envolvidas com a ação da deliberação moral: Decisão, Normativo, Compromisso, Conduta, Ação, Valor, Responsabilidade, Protocolo, Conhecimento, Atitude, Conflito e Dilema.

Os depoimentos foram obtidos por meio de entrevista fenomenológica autorizada, que possibilitou um encontro empático entre as pesquisadoras e as enfermeiras participantes, após assinatura do Termo de Consentimento Livre e Esclarecido (TCLE), com a possibilidade de desistência da participação em qualquer momento, sem nenhum tipo de ônus ou prejuízo. Em um ambiente de encontro, confortável, a entrevista foi dirigida por meio de um roteiro com as questões norteadoras: "Fale-me como você tem vivido a deliberação moral ou a tomada de decisão ética/moral no APHM?"; "Como você expressa suas expectativas frente à deliberação moral no APHM?"

A análise dos depoimentos foi processada por meio da leitura exaustiva e obtenção da essência do fenômeno. Para tal, foi realizada a etapa ideográfica em cinco momentos visando desvelar o vivido da ação de deliberar das participantes. No primeiro momento foi feito a transcrição na íntegra das falas com leitura vertical; posteriormente, em um segundo tempo, foram destacadas as locuções de efeito com descrição das unidades de contexto. No terceiro momento foi realizada nova redução fazendo aparecer o fenômeno da deliberação moral na sua essência. No quarto momento buscou-se as convergências das unidades de significado que expressaram motivos em comum referentes à ação, descritas por palavras, e, em seguida, foram construídas as categorias concretas na perspectiva de Schutz.

Emergiram as categorias concretas do vivido, como síntese objetiva dos diferentes significados da ação da deliberação moral das enfermeiras, denominada por Schutz como motivos existenciais. Os motivos "porque" foram expressos pelas experiências passadas das enfermeiras no cotidiano vivenciado no APH, que conduzem o agir no cenário do atendimento de rua. A discussão dos dados foi fundamentada no referencial teórico da fenomenologia social de Alfred Schutz e em literaturas relacionadas ao fenômeno em estudo.

\section{Resultados}

A população do estudo foram 12 enfermeiras, 7 mulheres e 5 homens, com idade compreendida entre 28 e 45 anos, com 2 a 17 anos de exercício profissional, com mais de um vínculo empregatício e cumprimento de cerca de 70 horas semanais. Havendo o predomínio do sexo feminino, utilizou-se a nomenclatura enfermeira para a identificação dos participantes do estudo.

Da análise dos depoimentos, para atender o objetivo de compreender como as enfermeiras vivenciam a ação da deliberação moral na sua prática cotidiana, emergiram três categorias concretas experienciadas no mundo-vida do APHM: expressando o contexto do APHM vivenciado como fundamento do ato de deliberar; revelando o conhecimento técnico-científico como fundamento para a ação da deliberação moral das enfermeiras no APHM; expressando a dimensão social das relações estabelecidas entre as equipes como fonte de problemas éticos no cenário do APHM.

\section{Expressando o contexto do APHM vivenciado como fundamento do ato de deliberar}

Nessa categoria, as enfermeiras expressam que, na concretude da existência vivida com outros atores no mundo social do APHM, há preocupação com os fatores externos ou ambientais e institucionais para a prática da deliberação moral. Entre estes, estão a falta de apoio da comunidade, a dependência do cenário encontrado, a ocorrência de situações adversas e a exposição em ser filmado pelo público.

\footnotetext{
A gente tem situações em que a própria comunidade se volta contra [...] os profissionais que estão prestando assistência [...] (Normativo).

[...] na verdade a nossa deliberação [...] muitas vezes depende de todo um cenário que a gente encontra, né? Para depois a gente chegar numa decisão [...] (Decisão).
} 
[...] a gente tem que ter muito cuidado quando a gente vai tomar alguma decisão [...] todo mundo boje tem uma camarazinha de vídeo e a gente fica exposto a tudo isso. (Responsabilidade).

\section{Revelando o conbecimento técnico- -científico como fundamento para a ação da deliberação moral das enfermeiras no APHM}

Para as enfermeiras, a deliberação moral é influenciada pela base de conhecimento adquirida com as suas experiências armazenadas. Esse conhecimento constitui-se no saber técnico-científico e no que estudou, em determinado momento, de acordo com o seu papel no serviço. A bagagem de conhecimento ajuda a responder às possibilidades de infrações éticas/deontológica perante o Conselho Regional de Enfermagem (COREN), como descrito nos depoimentos:

\footnotetext{
Ef fazer aquilo que the compete de acordo com o que você estudou, de acordo com o seu papel no serviço naquele momento, com o que você aprendeu com o outro [...] (Conduta).

Quando entrei no serviço as minhas dificuldades foram menores, o conbecimento técnico, e a busca pela aproximação com as situações de emergência colaborou para as minhas deliberações [...] eu acredito! (Responsabilidade).

Se você tem o conhecimento técnico-científico, você tem respaldo de [...] responder alguma coisa perante o COREN [...] (Protocolo).
}

\section{Expressando a dimensão social das relações estabelecidas entre as equipes como fonte de problemas éticos no cenário do APHM}

As enfermeiras, em seus depoimentos, descrevem o comportamento moral dos profissionais, bem como a existência de lacunas de socialidades entre essas profissionais, e da equipe de enfermagem com a equipe médica da regulação que atuam no serviço. Tais atitudes apresentam-se como potencializadoras de problemas éticos no cenário do APHM.

A gente tem questões relacionadas à personalidade das pessoas, a vícios das pessoas, a descompromissos das pessoas que estão fazendo parte da equipe [...] a tentar uniformizar o trabalbo da equipe [...] decisão para tomar assim é bastante difícil para o enfermeiro sendo que, envolve uma equipe [...] (Conflito).

[...] você trabalha em conjunto e pode acontecer que o grupo não esteja coeso, e uma ação que poderia ser completa e eficaz às vezes pode ser atrapalhada pela agilidade ou por uma pressa no atendimento [...] (Dilema).

Mas, às vezes a equipe não tá entrosada ou a situação realmente é adversa [...] Às vezes a gente não consegue comunicação com os médicos reguladores. (Valor).

As enfermeiras ainda destacam nas falas as relações de poder instituídas entre os membros das equipes do APHM, como observado nos depoimentos:

A gente toma aquela decisão, unidade de suporte avançado [...] aquela decisão baseada na conduta médica, né? O médico é quem geralmente determina a conduta [...] (Conduta).

Por mais que a gente tenha o conbecimento, a gente tem essa obrigação de entrar em contato com o médico regulador [...] (Protocolo).

[...] geralmente a gente não toma decisão sozinha a não ser aquela decisão que já está protocolada, em diversos protocolos de atendimento que a gente tem, fora isso, a gente só toma decisão acompanhada do médico [...] porque já está no protocolo a gente pode fazer, né? A gente é preso, né? (Atitude).

\section{Discussão}

Para as enfermeiras do estudo, desvelar a deliberação moral foi descortinar relações sociais estabelecidas em um cenário descrito por exposição a condições de trabalho que podem ser geradores de sentimentos, como angústia, frustração e sofrimento. Esses sentimentos podem associar-se à sensação de impotência para um agir em situações que remetem à necessidade frequente de deliberação moral ${ }^{(10)}$.

Em consonância, diante de limites ou obstáculos no cuidado, o distresse moral pode ser vivenciado pelas enfermeiras, pois abrange um processo de experiências individuais em que interagem as questões éticas, os problemas, as incertezas, a sensibilidade e a deliberação moral dessas profissionais ${ }^{(11)}$.

Durante o atendimento, os profissionais que compõem as equipes do APHM ficam expostos a alguns fatores que dificultam o processo de trabalho, como riscos ambientais, acidentes em rodovias, violência e lugares de difícil acesso, envolvimento da população como estressores que provocam adoecimento, bem como a ocorrência de comunicação ineficaz entre os profissionais $^{(12)}$. 
O comprometimento da privacidade foi destacado em função das exposições constantes com filmagens da população que se encontra no cenário de rua. No entanto, a sensação de identidade, espaço pessoal e dignidade, considerada como privacidade, precisa ser respeitada no momento dos atendimentos de urgência e emergência $^{(7)}$. Neste estudo é perceptível que esta questão ética influencia a tomada de decisões dos envolvidos no pré-hospitalar, impactando na deliberação sobre os fatos.

Reconhecer os fatos relacionados ao atendimento em si e os valores que carrega a construção individual, social, cultural e histórico é essencial para o percurso deliberativo ${ }^{(4)}$. Assim, o estar em atendimento com um paciente grave em rua faz emergir problemas éticos constantes que exigem ponderação das decisões tomadas pelas equipes.

Nesses cenários, valores essenciais podem ser implicados nas tomadas de decisão, como a beneficência, a dignidade humana, a supremacia da vida, a justiça e a responsabilidade profissional $^{(13)}$.

Vale ressaltar ainda que no mundo social o homem vivencia uma situação biográfica determinada, se situa de maneira específica no mundo da vida, e é nesse contexto que pensa, sente e age ${ }^{(9)}$.

Os depoimentos apresentados neste estudo desvelaram que, no ato das relações sociais estabelecidas e nas motivações, o compartilhamento de conhecimento subsidia o agir das enfermeiras no cenário do APHM. Compartilham um mundo-vida existente, com profissionais do serviço, em busca de saberes diversos para a prática de cuidados nos cenários de rua.

Assim, no mundo-vida do APHM, a interpretação pode ser baseada sobre um estoque de experiências prévias a seu respeito; a bagagem de conhecimento, as próprias experiências e aquelas que são transmitidas operam como uma fonte de referência ${ }^{(4)}$.

Nesse contexto, enfermeiras iranianas convergem com os depoimentos quando destacam que as missões anteriores e a convivência com colegas experientes desempenham um papel importante na condução dos desafios éticos ${ }^{(7)}$. Novos valores podem ser sedimentados em vigor de uma prática profissional qualificada.

Em um estudo americano, enfermeiras do serviço de ambulâncias compreendem ainda $\mathrm{O}$ julgamento clínico e o conhecimento científico como ferramentas indispensáveis para a tomada de decisão e realização de práticas seguras ${ }^{(14)}$.

No Brasil, a inserção de enfermeiros treinados e habilitados no APH possibilita qualificação e segurança assistencial à população em situação de urgência, impactando na qualidade do cuidado ofertado com melhores resultados para o sistema de saúde ${ }^{(15)}$.

É fundamental, assim, destacar a necessidade de estratégias de qualificação das equipes como processo permanente, inserido no cotidiano das ações do serviço, tendo em vista o espaço para a educação dentro da rotina do trabalho, como previsto no Regulamento Técnico dos Sistemas Estaduais de Urgência e Emergência, da Portaria n. 2048, do Ministério da Saúde ${ }^{(16)}$. Esta normativa deixa demarcada as estratégias possíveis para a inserção da prática deliberativa que faz congruência com a autoavaliação profissional e a autoeducação necessárias para a condução dos problemas éticos que emergem nos cenários práticos ${ }^{(4)}$.

Considera-se a deliberação moral como um método que realiza análise dos eventos da vida, dos acontecimentos históricos e culturais, corroborando a explicação, argumentação e justificativa das eleições morais ${ }^{(17)}$. A condução de espaços educativos, dialógicos, no APHM, pode ser um balizador de identificação de problemas éticos, bem como das estratégias para as decisões éticas.

Compreende-se pelas falas, que a atuação das enfermeiras nas Unidades Móveis não pode ser vista como isolada no mundo da vida, visto que há uma rede de relacionamentos instituída que se torna definidora de suas ações. As dificuldades expressadas nas relações sociais mantidas revelam as posições ocupadas pelas profissionais do serviço ao exercerem funções pré-estabelecidas distintas.

A existência frequente de uma relação indireta entre as enfermeiras e os demais membros 
da equipe que atuam no processo do cuidar no APHM é propiciadora de conflitos devido ao processo de decisão rotinizado, que pode retirar a instituição de um espaço dialógico entre os profissionais $^{(10)}$.

Estudo sobre os entraves enfrentados no SAMU, na percepção de enfermeiras, apresenta também os entraves existentes na comunicação entre os profissionais da Intervenção e Regulação como fatores que dificultam o trabalho do SAMU, corroborando uma minimização da qualidade da assistência que tem como ordenador um chamado iniciado com o atendimento de profissionais de uma Central de regulação, que não se encontram na cena da ocorrência ${ }^{(12)}$. Percebe-se, assim, uma relação que se caracteriza pelo anonimato entre os agentes sociais oriundos da Regulação e da Intervenção responsáveis pelo cuidado no APHM, além do reconhecimento dessa situação como relações distantes para a realização dos atendimentos.

Sendo assim, nessa área, em que a cooperação é fundamental para a qualidade $e$ a segurança dos cuidados prestados, as dificuldades no relacionamento interpessoal certamente comprometem o trabalho da equipe e o repertório de opções para uma deliberação prudente $^{(5)}$.

Configura-se a existência de grupos sociais no APHM que se rompem, mantendo as relações de dominados e dominantes, com regras impostas, dificultando a horizontalização das relações e proporcionando um distanciamento das relações "eu-tu, eu-nós", o que pode influenciar no despertar de uma consciência moral no momento de deliberar no cenário APHM.

As relações das equipes, quando provocam a continuidade, na prática, de um encontro hierarquizado, com verticalização e infantilização das relações, rompe o diálogo e a criatividade entre os profissionais da saúde ${ }^{(18)}$.

Tal fato pode transgredir as ações de deliberação, potencializar os problemas éticos, possibilitando apenas uma decisão racional, rápida e pouco refletida, mesmo sabendo que existem situações no cotidiano dessas profissionais que não estão listadas como regras prontas protocoladas, inclusive as que permeiam o ato de deliberar.

Assim, percebe-se como contraditórias as relações sociais construídas, pois à medida que se propõe uma homogeneidade à equipe do SAMU com a padronização de um mesmo uniforme, rompe-se a horizontalização e o equilíbrio destas relações quando emerge a necessidade de demarcação de territórios profissionais no mundo cotidiano do fazer ${ }^{(19)}$. Apreende-se, nesse contexto, a ausência de reciprocidade e intersubjetividade entre as equipes que ocupam o espaço do APHM.

Oriundo de um modelo biomédico, assistencialista, centrado na figura do profissional médico, o atendimento móvel carrega a origem de um serviço conduzido por protocolos ${ }^{(5)}$. O vivido em um mundo com regras impostas, no qual os elementos contraditórios e incoerentes não são mobilizados, evita que o indivíduo transcenda os requisitos necessários de seus planos e operações práticos, os quais tendem a assumir um caráter rotineiro $^{(9)}$.

Entretanto, propõe-se um rompimento de paradigma com a produção de saúde baseada não somente no resultado técnico-material, mas pautada nos valores, relações e afetos que proporcione uma troca inter-humana de caráter horizontal, coletivo-cooperativo ${ }^{(18)}$.

Assim, pertencente a um cotidiano com interesses comuns, esse tornar consciente do modo de deliberar das enfermeiras do APHM projeta um movimentar-se, mediante reflexões das relações cotidianas desse grupo social, com possibilidades para um agir que conduza o exercício da autonomia, a transformação de valores no momento da prática deliberativa, com o refazimento diário do exercício profissional.

A prática deliberativa no campo da bioética possibilita um espaço para debate entre ética, poder e emancipação para um lócus de práticas equitativas e democráticas como via de ponderação das decisões ${ }^{(20)}$.

No entanto, nas perspectivas das enfermeiras que vivenciam a deliberação moral, emerge um cenário concreto, conflituoso, que consagra uma distância dos atores sociais envolvidos no 
APHM, descritos como profissionais atuantes nas unidades móveis e Central de Regulação das Urgências (CRU), propiciando a ocorrência de problemas éticos nas relações de equipes.

O pensamento schutziano estabelece a necessidade de uma relação face a face oriunda das relações sociais e intersubjetividades, com simultaneidade direta entre uma e outra pessoa ${ }^{(6)}$. No entanto, fica estabelecida, no cotidiano descrito pelas participantes, uma relação de anonimato, de distanciamento, que, quando em grau máximo, considera o outro como número ou apenas função ${ }^{(9)}$.

Foi possível uma identificação da realidade de significados, razões e justificativas para a deliberação dessas profissionais no seu mundo cotidiano. Ciente que as enfermeiras podem ser sujeitos do seu próprio mundo-vida, o ponto de partida para essa ação pode ser uma avaliação dos problemas éticos vivenciados, abrindo-se um leque de possibilidades para a sua resolução prudente, com um repertório de ações fundamentadas em uma relação interacional entre as equipes, intervenção, regulação e coordenação (5)

Assim, a necessidade do exercício de práticas deliberativas pode indicar o uso sistematizado de métodos que possam favorecer o desenvolvimento de habilidades e competências, para uma excelência do agir ético no cenário do APHM, que particularize profissionais da intervenção e da regulação, e, ao mesmo tempo, possa fortalecer o diálogo entre os pares e a minimização de ocorrência de distresse moral entre as enfermeiras pertencentes a este mundo-vida.

Como limitação deste estudo, que desvela o mundo-vida de um grupo social, tem-se a participação de um grupo específico de uma área metropolitana e que pode divergir dos significados de vivências de outros atores sociais marcados por tempos, histórias e motivações distintas na prática deliberativa no APHM.

\section{Conclusão}

O estudo possibilitou uma aproximação com o mundo-vida de enfermeiras do APHM, cenário de atuação e de deliberação diante das questões éticas vivenciadas, com o registro de um cotidiano demarcado por rompimentos nas relações que se refletem em limites. No entanto, também revela elementos que podem favorecer a prática deliberativa, como as experiências do vivido, o compartilhamento das situações e os conflitos que demandam a tomada de decisão.

O estudo faz emergir a proposta de pautar o exercício da deliberação moral como um método prático no mundo-vida do APHM tão relevante como os treinamentos de equipe, favorecendo um cenário relacional, deliberativo, dialógico e próximo de uma relação "eu-tu-nós".

Estudar a deliberação moral com base nas ações passadas desse grupo social faz imperar um novo olhar para a prática das enfermeiras, que motive um agir prudente, com escolhas eficazes, conciliadas com práticas seguras e éticas.

Não havendo esgotamento, propõe-se novas abordagens visando a ampliação dessa temática, para que sejam oferecidos subsídios que provoquem um despertar da consciência moral, modifiquem as ações de deliberar das enfermeiras no exercício profissional e estabeleça relações horizontais entre os atores sociais que fazem parte do cenário do APHM mediante novas motivações que conduzam as ações de deliberar.

\section{Colaborações:}

1 - concepção, projeto, análise e interpretação dos dados: Simone da Silva Oliveira, Adriana Brait Lima e Darci de Oliveira Santa Rosa;

2 - redação do artigo e revisão crítica relevante do conteúdo intelectual: Simone da Silva Oliveira, Adriana Brait Lima, Darci de Oliveira Santa Rosa, Genival Fernandes de Freitas e Mariana Oliveira Antunes Ferraz;

3 - aprovação final da versão a ser publicada: Simone da Silva Oliveira, Genival Fernandes de Freitas e Mariana Oliveira Antunes Ferraz.

\section{Referências}

1. Brasil. Ministério da Saúde. Portaria de Consolidação n. 3, de 28 de setembro de 2017. Consolidação das normas sobre as redes do 
Sistema Único de Saúde [Internet]. Diário Oficial da União. Brasília (DF); 2017 [cited 2020 Aug 12]. Available from: http://bvsms.saude.gov.br/bvs/ saudelegis/gm/2017/prc0003_03_10_2017.html

2. Dolor ALT, Freitas GF, Oguisso T. Aspectos Éticos-Legais em face das situações de ocorrências éticas no contexto do Atendimento Pré-Hospitalar (APH). In: Freitas GF, Oguisso T. Ética no contexto da prática de Enfermagem. Rio de Janeiro: Medbook; 2010. p. 219-53.

3. Nora CRD, Schaefer R, Alvaréz EJS, Ramos MDB. Sensibilidade moral e fatores relacionados: percepção de enfermeiros. Cogitare Enferm. 2016;21(4):1-8. DOI: 10.5380/ce.v21i4.47410

4. Zoboli E. A aplicação da deliberação moral na pesquisa empírica em bioética. Rev iberoam bioét. 2016;2:1-19. DOI: 10.14422/rib.i02.y2016.006

5. Oliveira SS, Santos DV, Zoboli E, Santa Rosa DO. Deliberação moral da enfermeira no cuidado em atendimento pré-hospitalar. In: Vale EG, Peruzzo SA, Felli VEA, organizadoras. PROENF Programa de Atualização em Enfermagem. Gestão: Ciclo 6. Porto Alegre: Artmed; Pan-americana; 2017. p. 33-55.

6. Schutz A. Estudios sobre teoria social: escrito II. Buenos Aires: Amorrortu; 2003.

7. Tobori M, Borhani F, Abbaszadeh A, Atashzadeh-Shoorideh F. Experiences of pre-hospital emergency medical personnel in ethical decisionmaking: a qualitative study. BMC Med Ethics 2018;19(95):1-9. DOI: 10.1186/s12910-018-0334-x

8. Anjos MS, Oliveira SS, Santa Rosa DO. Perspectivas de Enfermeiras em Atendimento Pré-hospitalar Móvel. Rev baiana enferm. 2016;30(1):375-81. DOI: $10.18471 /$ rbe.v1i1.14442

9. Schutz A. Sobre a fenomenologia e relações sociais. Rio de Janeiro: Vozes; 2012.

10. Oliveira SS. Deliberação moral da enfermeira no cuidado pré-hospitalar à luz da fenomenologia social [dissertação]. Salvador (BA): Universidade Federal da Bahia; 2014.

11. Barth PO, Ramos FRS, Barlem ELD, Dalmolin GL, Schneider DG. Validation of a moral distress instrument in nurses of primary health care. Rev Latino-Am Enfermagem. 2018;26:e3010. DOI: 10.1590/ 1518-8345.2227.3010
12. Mata KSS, Ribeiro IAP, Pereira PSL, Nascimento MVF, Carvalho GCN, Macedo JB, et al. Obstacles in SAMU pre-hospital care: nurses' perception. Rev enferm UFPE online. 2018;12(8):2137-45. DOI: 10.5205/1981-8963-v12i8a236537p2137-2145-2018 Portuguese

13. Albuquerque A, Oliveira AA. Manual de Direitos humanos para a Enfermagem. Brasília: UniCEUB; COFEN; 2016 [cited 2020 Oct 14]. Available from: http://biblioteca.cofen.gov.br/ manual-de-direitos-humanos-para-enfermagem/

14. Sjölin H, Lindström V, Hult $H$, Ringsted C, Kurland L. What an ambulance nurse needs to know: a content analysis of curricula in the specialist nursing programe in prehospital emergency care. Int Emerg Nurs. 2015;23(2):127-32. DOI: $10.1016 /$ j.ienj.2014.09.002

15. Malvestio MAA, Beringer LPB, Martuch SD, Fonseca MAS, Silva L, Souza EF, et al. Enfermagem em práticas avançadas no atendimento pré-hospitalar: oportunidade de ampliação do acesso no Brasil. Enfer Foco [Internet]. 2019 [cited 2020 Oct 14];10(6):157-64. Available from: http://revista. cofen.gov.br/index.php/enfermagem/article/ view/2594/666

16. Brasil. Ministério da Saúde. Portaria n. 2048, de 5 de novembro de 2002. Aprova o Regulamento Técnico dos Sistemas Estaduais de Urgência e Emergência [Internet]. Diário Oficial da União. Brasília (DF); 2002 [cited 2020 Aug 12]. Available from: https://bvsms.saude.gov.br/bvs/saudelegis/ gm/2002/prt2048_05_11_2002.html

17. Zoboli ELCP. Bioética clínica na diversidade: a contribuição da proposta deliberativa de Diego Gracia. Bioethikos [Internet]. 2012 [cited 2020 Sep 7];6(1):49-57 Available from: http://www. saocamilo-sp.br/pdf/bioethikos/91/a05.pdf

18. Gomes D, Zoboli E, Finkler M. Problemas éticos na saúde bucal no contexto da Atenção Primária a Saúde. Physis. 2019;29(2):e290208. DOI: 10.1590/ S0103-73312019290208

19. Velloso ISC, Araujo MT, Nogueira JD, Alves M. Gerenciamento da diferença: relação de poder e limites profissionais no serviço de atendimento móvel de urgência. Rev Enf Ref. 2014;serIV(2):71-9. DOI: http://dx.doi.org/10.12707/RIII1374 
20. Feito L. Pensar en bioética: mejor com tempo. Bioétic complut. 2017 [cited 2020 Sep 6];32:2. Available from: https://www.ucm.es/data/cont/ docs/137-2017-12-21-Revista\%2032.pdf
Recebido: 11 de setembro de 2020

Aprovado: 15 de outubro de 2020

Publicado: 23 de novembro de 2020

A Revista Baiana de Enfermagem utiliza a Licença Creative Commons - Atribuição-NãoComercial 4.0 Internacional. https://creativecommons.org/licenses/by-nc/4.0/ Este artigo é de acesso aberto distribuído sob os termos da Licença Creative Commons (CC BY-NC). Esta licença permite que outros remixem, adaptem e criem a partir do seu trabalho para fins não comerciais. Embora os novos trabalhos tenham de lhe atribuir o devido crédito e não possam ser usados para fins comerciais, os usuários não têm de licenciar esses trabalhos derivados sob os mesmos termos. 